Research article

\title{
Phytoecological studies of some protected and degraded forest areas of lowland humid forest, Ondo state, Nigeria: a comparative approach
}

\author{
Iyagin F. O.* and Adekunle V. A. J. \\ Department of Forestry and Wood Technology, Federal University of Technology Akure, \\ P.M.B 704, Ondo State, Akure, Nigeria
}

*Corresponding Author: fiyagin@yahoo.com

[Accepted: 24 December 2017]

\begin{abstract}
This study compared the phytoecological characteristics of some protected areas [Strict nature reserve (SNR), Oluwa natural forest (ONF) and permanent sample plot 29 aimed at biodiversity conservation and a degraded forest not under reservation] in the lowland humid forest, Ondo state, Nigeria. Data were collected using Systematic Line Transect method with two parallel transects of $200 \mathrm{~m}$ apart for plot location. Four sample plots $(25 \mathrm{~m} \times 25 \mathrm{~m})$ were laid alternately on each transect in each of the forests. In each plot, trees with $\mathrm{DBH} \geq 10 \mathrm{~cm}$ were measured (diameters at the base, middle, top and total height), identified and classified into families and frequency of occurrence to ascertain the present status of the protected areas in term of tree diversity, abundance and yield. A total of 411 stems.ha $^{-1}$ from 78 species and 30 families was recorded in the study sites. Celtis zenkeri, Cola gigantea and Funtumia elastica were common to all the forests. The DBH distribution curve of the species followed inverse J-shaped pattern with $49.4 \%$ of trees falling within the lowest diameter class $(10-20 \mathrm{~cm})$ while only $7.14 \%$ falls in the highest diameter class $(>80 \mathrm{~cm}$ ). SNR and ONF have emergent trees whose heights were above 30 $\mathrm{m}(18.7 \%$ and $7.02 \%$ of the total number of trees in the Sterculaceae and Meliaceae families, respectively) whereas $14.90 \mathrm{~m}$ was the highest height recorded in the free forest. On biovolume yield, ONF has the highest volume per hectare $\left(141.06 \mathrm{~m}^{3}\right)$ while the least $\left(14.56 \mathrm{~m}^{3}\right)$ was from the forest not under reservation. The Shannon-Weiner index, Pielou's Evenness index and Margalef's index for all the forest reserves are 5.11, 0.69 and 12.79 respectively with the highest for ONF. These indices indicated that the protected areas are mature and foster in-situ conservation of tree species especially the keystone species.
\end{abstract}

Keywords: Biodiversity - Protected areas - Conservation - Deforestation - Forest structure.

[Cite as: Iyagin FO \& Adekunle VAJ (2017) Phytoecological studies of some protected and degraded forest areas of Lowland Humid forest, Ondo state Nigeria: a Comparative approach. Tropical Plant Research 4(3): 496-513]

\section{INTRODUCTION}

Biodiversity has been defined as the variety and variability of life forms on earth. The "biological diversity" typically measures variation at the genetic, the species, and the ecosystem level, though not evenly distributed on earth but richest in the tropics (UNEP 2011). Flora and fauna diversity and the activities vary from one ecological zone to the other in the tropic. This could be attributed to the differences in climate and weather condition of the ecosystems. The tropical rainforest, which is located in the southwest and southeast geopolitical zones of Nigeria, is highly complex and is known for its astonishing wealth of plant species because of the usual favorable climate (Adekunle et al. 2007). Forest biodiversity provides wide variety of goods and services that support the existence of humans on earth. These services include the provision of services which involve the production of renewable resources such as food, wood, fertile land, wildlife, and fresh water, regulating services. These are those that are responsible for environmental changes (e.g. climate regulation and mitigate, biodiversity conservation, combating desertification encroachment, pest/disease control) and cultural services 
representing human values and beliefs (e.g. landscape aesthetics, cultural heritage, outdoor recreation and spiritual significance) (Behera et al. 2012, Cardinale et al. 2012, Daniel et al. 2012). According to Phillips et al. (2003) and Royal Society (2003), biodiversity assessment was recognized by international policy process such as the convention on biological diversity, as inevitable tool to guide conversation. The importance of Biodiversity can be related to the phytosociology of a community (Bajpai et al. 2015).

As stated by Dengler (2017), Phytosociology is an aspect of science which study vegetation in terms of plant assemblages, classification and characterization of the vegetation types based on the floristic composition in the plant community. Phytosociological studies are essential to characterize and classify plant community (trees) in term of their structure and composition for protecting the natural plant environment and proper management of the forest resources. Understanding and appreciating the need for biodiversity conservation in Nigeria is of great worth (Aju \& Ezeibekwe 2010) as this could help in reducing the continuous external threat imposed on protected areas and the restoration of loss that has taken place in the ecosystems. More than half of Nigeria's primary forest has been lost to deforestation through: urbanization, over exploitation of timber, subsistence agriculture, and increase in the demand for fuel wood in the last decade (FAO 2006). Other factors against biological diversity conservation in the tropics include explosive growth in human population, poverty, and failure to implement the methods or approaches aimed at sustainable agriculture and forestry practices (Ekpo et al. 2011).

Protected Areas (PAs) are potentially beneficial for carbon sink and environmental conservation. Adekunle et al. (2014), defined Protected Areas as geographical space, recognised, dedicated and managed by means of legal or other effective strategies to achieve the continuing conservation of nature with associated ecosystem services and cultural values. Protected areas were categorised into: the Strict Nature Reserves (strict protection), National Parks (Ecosystem Conservation and Protection), Natural Monuments (conservation of natural features), Habitat/Species Management Area, Protected landscape/seascape (Landscape/Seascape conservation and recreation) and Managed Resource Protected Area (Sustainable use of natural resources) (IUCN 2008). Every PA, irrespective of the management strategy should have the following objectives: conservation of the composition, structure, function and evolutionary potential of biodiversity; contributions to regional conservation strategies; maintenance of diversity of landscape or habitat and of associated species and ecosystems; long-term maintenance of the specified conservation targets; maintain the values for which it was assigned in perpetuity; be operating under the guidance of a management plan and possess a clear and equitable governance system (IUCN 2008, Adekunle et al. 2014). Also, it should be able to conserve significant landscape features, geomorphology and geology; provide regulatory ecosystem services, including buffering against the impacts of climate change and recreational benefits; provide for cultural, spiritual, educational opportunities and scientific research purposes and deliver benefits to resident and local communities." These losses are unfavourable to the continued existence of animals, plants, human beings, and the general environment because biodiversity conservation is recognized as a global life support system (Isichei 1995).

Tree canopy of the tropical rainforest ecosystem is made up of the upper layer (at about 30-40 m), the second layer (between 23-30 m) and the lower layer which is made up of saplings of a number of species (Bourgeron 1983). However, the high species diversity and the notable number of goods and services obtainable from the rainforest ecosystem are partly responsible for the pressure to which it has been subjected for centuries; and is presently on the increase (Onyekwelu et al. 2005) thereby increasing deforestation and forest degradation. These, have been reported as the principal causes of forest cover change (Sedano et al. 2016) which is responsible for the high percentage of the global carbon emission today (Van der werf et al. 2009). Deforestation has been defined as the unchanging or long-term conversion of forest land use to other non-forest uses thereby, causing the sudden and rapid change in land cover; consequently resulting in forest degradation, contributing to the build-up of the carbon dioxide content in the atmosphere (GOFC-GOLD 2009, van der werf et al. 2009). Other direct drivers of deforestation include agricultural activities, infrastructure development and settlements (Halperin \& Turner 2013). Forest degradation has caused lots of havoc among which are; colossal reduction or total loss of forest land due to several human activities (Chadman 2008, Mackey et al. 2008, Simula 2009, FAO 2011) and reduction in forested landscape carbon stocks in relation to its natural carbon carrying capacity (Mackey et al. 2008). This research therefore established the current status of selected protected areas in the tropical rainforest ecosystem of southwest Nigeria that could enhance sustainable forest management ecosystem restoration, thereby increasing the biodiversity efforts of all forest stakeholders. 


\section{MATERIALS AND METHODS}

Study areas

This study was carried out at three PAs and an adjoining free forest area to one of the selected PAs (Fig. 1). The PAs are a Natural Strict Reserve within Akure Forest Reserve, Oluwa Forest Reserve and a Permanent Sample Plot (PSP 29). The free forest area is adjacent to the PSP 29.

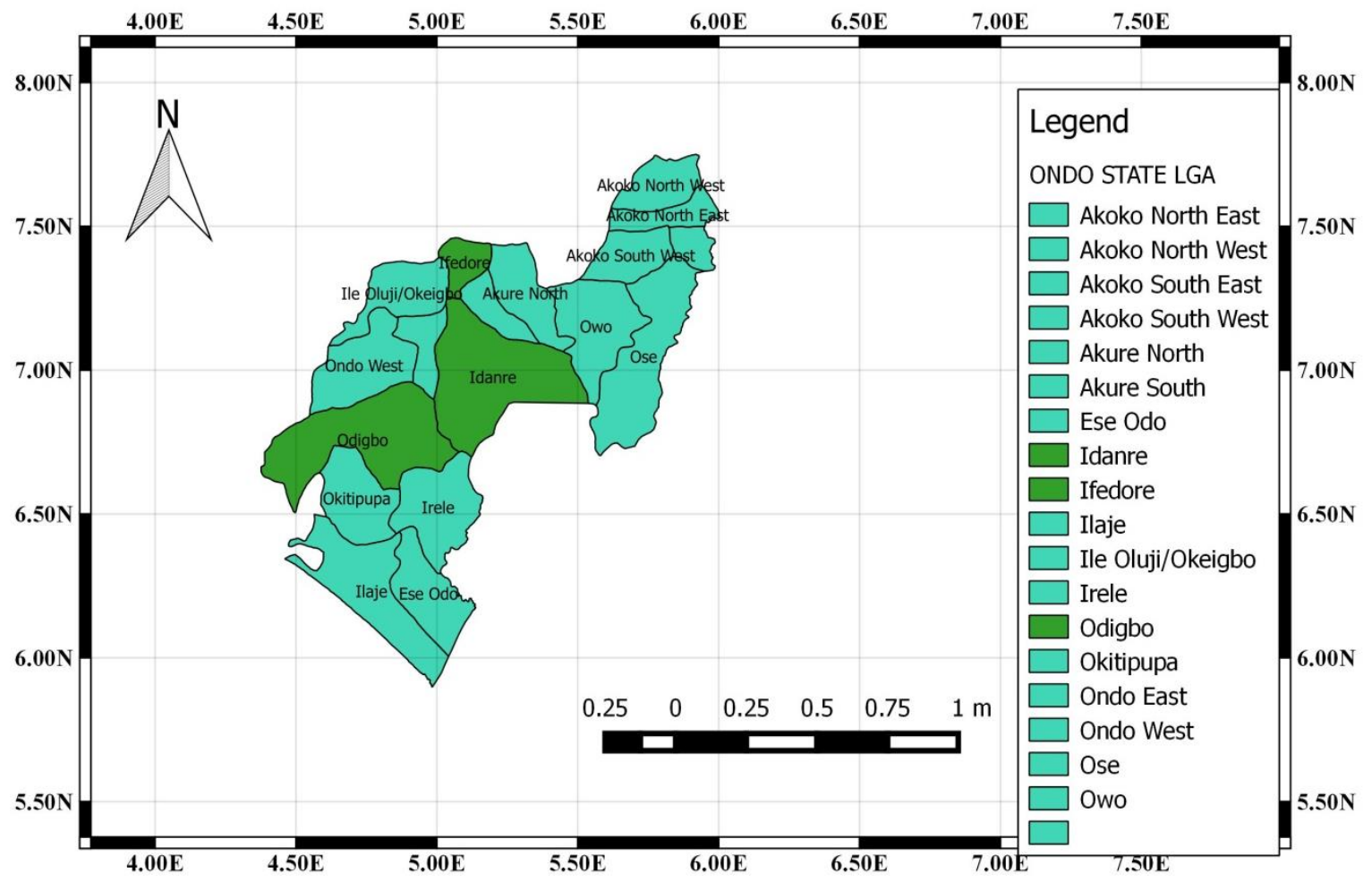

Figure 1. Map of Ondo state showing the location of the forest reserves.

Location, climate and vegetation

Akure Forest Reserve (Aponmu) is one of the Strict Nature Forest Reserves in Nigeria located in the tropical rainforest in Ondo State. It covers an area of about 32 hectares (Adeduntan \& Olusola 2013) and lies between latitude $06.59718^{\circ} \mathrm{N}$ and longitude $004.49199^{\circ} \mathrm{E}$. In this protected area, the mean annual rainfall is about 1700 $\mathrm{m}$ and the temperature ranges from $20.6-33.5^{\circ} \mathrm{C}$. Oluwa natural forest is within Oluwa Forest Reserve. This forest lies between latitude $06.59718^{\circ} \mathrm{N}$ and longitude $004.49199^{\circ} \mathrm{E}$ and covers an area of 87,816 ha (Adeduntan 2009). The average rain fall of this reserve is $1700 \mathrm{~mm}$, Relative Humidity of $80 \%$, an annual temperature of $26^{\circ} \mathrm{C}$ with an average elevation of $100 \mathrm{~m}$ (Adekunle \& Dafiwhare 2011). Akure/ Owena Permanent Sample plot (PSP 29) is situated along Akure-Ondo road and about $1 \mathrm{~km}$ away from Cocoa Research Institute of Nigeria (CRIN) Owena substation. It covers an area of $65.93 \mathrm{~km}$ and falls within the high forest Zone of Nigeria between longitude $005.02911^{\circ} \mathrm{E}$ and latitude $07.20109^{\circ} \mathrm{N}$ (Pelemo et al. 2011). In this reserve, the relative humidity during the raining season ranges between 85 and $100 \%$ but less than $60 \%$ during the dry season (Fasinmirin \& Oguntuase 2008). It has a mean annual rainfall between (1300-1600 mm) and average temperature of $27^{\circ} \mathrm{C}$. This area was demarcated and being managed by the Federal Research Institute of Nigeria (FRIN).The Raining season in these locations starts from March and ends November with dry season starting from December and ends in February. The soil in these PAs is typical of the soil found in the rainforest region of the south western part of Nigeria. The soil texture is sandy-loamy but gradually becomes heavier in depth. The ferric luvisol soils are formed as a result of continuous weathering of the crystalline rock which feature mostly in the typical upland soils in many parts of South-Western Nigeria (FAO 1988).

\section{Data collection}

I. Plot demarcation: The systematic line transects was employed in laying of plots for data collection, two parallel transects of $200 \mathrm{~m}$ apart were laid in each of the study sites after a 50 meter distance has been taken from the edge of each forest. Thereafter, four sample plots of equal size $(25 \mathrm{~m} \times 25 \mathrm{~m})$ were laid alternately on each transect.

II. Measurement of tree growth variables: All trees with Diameter at Breast Height (DBH) $\geq 10 \mathrm{~cm}$ encountered in each sample plot were tagged identified and measured (Okali \& Ola-Adams, 1987, Chavan \& Rasal 2010). The diameters at the base, middle and top and the total height of all the trees $(\mathrm{DBH} \geq 10 \mathrm{~cm})$ in 
each plot were measured.

III. Tree species identification: The scientific names of all the tree species encountered on each field plot were recorded. Local names were used for tree species whose scientific names were not known immediately on the field and their parts (such as leaves, barks and fruits) were collected and taken to the herbarium for identification. Such species were temporarily referred to as unknown but were subsequently assigned their scientific name immediately after identification. All the species were classified into families and their frequency of occurrence were obtained to ascertain tree species diversity and abundance. The trees were also grouped into diameter distribution classes based on the DBH measurement taken on the field.

\section{Data analysis}

I. Basal Area computation: The total basal area for each of the sample plot was obtained by summing up all the Basal Area of the individual trees in the plots while the basal area per hectare was obtained by multiplying the mean Basal Area per plot with 16 (the number of $25 \mathrm{~m} \mathrm{X} 25 \mathrm{~m}$ plots in one hectare).

$$
B A=\frac{\pi D^{2}}{4}
$$

Where, $\mathrm{BA}=$ Basal area $\left(\mathrm{m}^{2}\right), \mathrm{D}=$ Diameter at breast height $(\mathrm{cm})$ and $\pi=3.142$ or $22 / 7$

II. Volume Estimation: The volume of individual trees was estimated using the Newton formula (Husch et al. 2003). Volume per hectare was obtained by multiplying the mean volume per plot with the number of $25 \mathrm{~m} \times 25$ $\mathrm{m}$ plots in a hectare (16).

$$
V=\frac{\pi h}{24}\left(D_{b}{ }^{2}+4 D_{m}{ }^{2}+D_{t}^{2}\right)
$$

Where, $V=$ Volume of tree $\left(\mathrm{m}^{3}\right), \mathrm{D}_{\mathrm{b}}=$ Diameter at the base $(\mathrm{cm}), \mathrm{D}_{\mathrm{m}}=$ Diameter at the middle $(\mathrm{cm}), \mathrm{D}_{\mathrm{t}}=$ Diameter at the top $(\mathrm{cm}), \mathrm{H}=$ height $(\mathrm{m})$

III. Biodiversity Indices and Tree Species Classification:

a) Species relative density was computed following Brashears et al. (2004)

$$
R D=\frac{n_{i}}{N} \times 100
$$

Where, $\mathrm{RD}(\%)=$ species relative density; $\mathrm{n}_{\mathrm{i}}=$ number of individuals of species $\mathrm{i} ; \mathrm{N}=$ total number of all tree species in the entire community

b) Species relative dominance (RDo (\%)) was computed using Aidar et al. (2001) equation:

$$
R D_{o}=\frac{\sum B a_{i} \times 100}{\sum B a_{n}}
$$

Where: $\mathrm{Ba}_{\mathrm{i}}=$ basal area of individual tree belonging to species $\mathrm{i}$ and $\mathrm{Ba}_{\mathrm{n}}=$ stand basal area.

c) The maximum diversity index was determined using the Shannon-Wiener diversity index (Kent \& Coker 1992, Guo et al. 2003). This was because it takes into account the richness and abundance of each species in different ecosystems (Price 1997).

$$
H^{\prime}=-\sum_{i=1}^{S} p_{i} \ln \left(p_{i}\right)
$$

Where, $H^{\prime}=$ Shannon diversity index, $S=$ the total number of species in the community, $p_{i}=$ proportion $S$ (species in the family) made up of the $i^{\text {th }}$ species and $\mathrm{Ln}=$ natural logarithm.

d) To determine the Species evenness (E), in each community, Shannon's equitability equation was adopted (Kent \& Coker 1992):

$$
E_{H}=\frac{H^{\prime}}{H_{M a x}}=\frac{\sum_{i=1}^{S} P_{i} \ln \left(P_{i}\right)}{\ln (S)} .
$$

e) Importance Value Index (IVI): The Importance Value Index for each species were obtained by summing up the RD and RDo divided by 2 (RD x RDo/2) (Brashears et al. 2004). This was used to express the share of 
each species in the tree community.

f) Family Importance Value (FIV): This is the sum of the relative dominance (RDm), relative density (RD) and relative frequency $(\mathrm{RF})$.

$\mathrm{RDm}=($ Total Basal area for a family $\div$ Total Basal area of all families $)$ X 100

$\mathrm{RD}=($ Number of individual ' $\mathrm{a}$ ' of family $\div$ Total number of all individual $) \mathrm{X} 100$

$\mathrm{RF}=($ Frequency ' $\mathrm{a}$ ' of family $\div$ Sum frequencies all of s families $) \times 100$

Therefore, the FIV is calculated as: RDm+ RD+ RF

Number 1 of Hill diversity index

$$
\begin{gathered}
\mathrm{N}_{1}=\exp \left(-\Sigma \mathrm{p}_{\mathrm{i}}\left(\ln \mathrm{p}_{\mathrm{i}}\right)\right) \\
\mathrm{pi}=\mathrm{n}_{\mathrm{i}} / \mathrm{N} \ldots \ldots \ldots \ldots . .
\end{gathered}
$$

Where: $\mathrm{p}_{\mathrm{i}}$ : is the proportional abundance of $\mathrm{i}^{\text {th }}$ species, $\mathrm{n}_{\mathrm{i}}$ : number of individuals of $\mathrm{i}^{\text {th }}$ species, $\mathrm{N}$ : total number of individuals.

Number 2 of Hill diversity index

$$
\begin{array}{r}
\mathrm{N}_{2} \text { : Reciprocal of Simpson's dominance Index; } \\
\mathrm{N}_{2}=1 / \Sigma \mathrm{p}_{\mathrm{i}}^{2} \ldots \ldots \ldots \ldots \ldots \ldots \ldots \ldots \ldots \ldots \ldots \ldots \ldots \ldots \ldots \ldots \ldots \ldots \ldots \ldots \ldots \ldots \ldots \ldots \ldots \ldots \ldots \ldots
\end{array}
$$

g) Shannon max diversity index was also determined using

$$
\mathrm{Hmax}=\mathrm{LnS}
$$

Where, $\mathrm{S}=$ the total number of species in the community

h) Sorensen's species similarity index was used to compare species diversity among the selected sites:

$$
\mathrm{SI}=\frac{2 C}{a+b+c+d} \times 100
$$

Where, $\mathrm{C}$ is the total number of species in four communities (i.e. aggregate of all species encountered in the entire study area); while a, b, c, \& d are the number of species at communities $1,2,3 \& 4$ respectively.

\section{RESULTS}

Tree species diversity and abundance

This study revealed that Oluwa Forest Reserve has the highest number of species ha ${ }^{-1}\left(170\right.$ stems.ha $\left.^{-1}\right)$ distributed into 23 and 54 families and species respectively. Monodora myristica (Gaertn.) Dunal, of Annonaceae family has the highest number of stem per hectare (19 spp.ha ${ }^{-1}$ ) with a relative density of 11.18 . This is followed by Buchholzia coriacea Engl.of Capparaceae family with 13 spp.ha ${ }^{-1}$ and relative density of 7.65 and Diospryos dendo Welw. ex Hiern (11 stems.ha $\left.{ }^{-1}\right)$ with relative density of 6.47. In Permanent Sample Plot 29, Celtis zenkeri Engl. of Ulmaceae family has the highest number of stems (15 stems.ha $\left.{ }^{-1}\right)$ and next in abundance is Sterculia rhinopetala K. Schum, of the family Sterculiaceae, and Funtumia elastica (Preuss) Stapf of Apocynaceae family (14 spp.ha ${ }^{-1}$ and 12 spp.ha ${ }^{-1}$ respectively) in the Strict Nature Reserve of Akure forest reserve. The tree species (Celtis zenkeri Engl., Cola gigantean A.Chev. and Funtumia elastica (Preuss) Stapf) are common to all the four forest sites assessed.

The tree species diversity is represented in table 1 . The Strict Nature Reserve has a total of 88 stems.ha $^{-1}$, distributed among 25 species and 15 different families. Sterculia rhinopetala K. Schum, has the highest number of stems (14 stems.ha $\left.{ }^{-1}\right)$. This is followed closely by Funtumia elastica (Preuss) Stapf (12 stems.ha $\left.{ }^{-1}\right)$. A total of 99 stems.ha $^{-1}$ which spans through 27 different species from 14 families were obtained at the PSP 29. In this location, Celtis zenkeri Engl. has the highest occurrence (15 stems.ha ${ }^{-1}$ ) and Relative density of 15.15 . The free forest area adjoining PSP 29 has a total of 54 stems.ha $^{-1}$. The most abundant species in the free area is Celtis zenkeri Engl. (11 stems.ha ${ }^{-1}$ ) with relative density of 20.37 . The total number of families and species that were encountered in the site is 12 and 23 respectively. From these results, about $39 \%$ of all the species were represented by single individual per ha.

The Families Importance Value for the selected forests is presented in table 2. The results revealed that 30 different trees families were encountered in the four forest types. Sterculiaceae family has the highest Family Importance Value (FIV) of $39.10 \%$. This was followed by Ulmaceae $(24.93 \%$ ) while Ochnaceae family has the least Family Importance Value of $0.49 \%$. The highest volume per hectare was also recorded for Papilionoideae family $\left(3.14 \mathrm{~m}^{3}\right)$, followed by Myristicaceae family $\left(2.92 \mathrm{~m}^{3}\right)$ and the least by Guttiferae family $\left(0.10 \mathrm{~m}^{3}\right)$. The highest RD and RDo were recorded for Sterculiaceae (19.46\%) and Bignoniaceae $(0.13 \%)$. 
Table 1. Tree Species abundance per ha, diversity indices and tree growth variables of the selected forest reserves.

\begin{tabular}{|c|c|c|c|c|c|c|c|c|c|c|c|c|}
\hline Sites & S.N. & Family & Species & nha $^{-1}$ & $\begin{array}{r}\text { MDBH } \\
(\mathbf{c m}) \\
\end{array}$ & $\begin{array}{r}\mathbf{H t} \\
(\mathbf{m})\end{array}$ & $\begin{array}{r}\mathbf{B A} \\
\left(\mathbf{m}^{\mathbf{2}}\right)\end{array}$ & $\begin{array}{r}\text { VOL } \\
\left(\mathbf{m}^{\mathbf{3}}\right) \\
\end{array}$ & PiLnPi & RD & $\begin{array}{r}\text { RDO } \\
(\%) \\
\end{array}$ & IVI \\
\hline \multirow[t]{25}{*}{ SNR } & 1 & Annonaceae & Monodora myristica (Gaertn.) Dunal & 3 & 22.03 & 22.43 & 0.05 & 0.34 & -0.12 & 3.41 & 1.35 & 2.30 \\
\hline & 2 & Apocynaceae & Alstonia boonei De Wild. & 1 & 16.40 & 15.00 & 0.02 & 0.20 & -0.05 & 1.14 & 0.61 & 0.34 \\
\hline & 3 & Apocynaceae & Funtumia elastica (Preuss) Stapf & 12 & 22.38 & 20.90 & 0.07 & 0.43 & -0.27 & 13.64 & 1.93 & 13.15 \\
\hline & 4 & Burseraceae & Canarium schweinfurthii Engl. & 5 & 59.24 & 33.62 & 0.31 & 2.71 & -0.16 & 5.68 & 8.90 & 25.29 \\
\hline & 5 & Ebenaceae & Diospyros mespiliformis Hochst. & 1 & 22.00 & 15.30 & 0.04 & 0.29 & -0.05 & 1.14 & 1.09 & 0.62 \\
\hline & 6 & Euphorbiaceae & Macaranga hurifolia Beille & 1 & 17.60 & 29.00 & 0.02 & 0.22 & -0.05 & 1.14 & 0.70 & 0.40 \\
\hline & 7 & Lecythidaceae & Petersianthus macrocarpum (P. Beauv) & 3 & 55.87 & 31.53 & 0.25 & 1.93 & -0.12 & 3.41 & 7.26 & 12.37 \\
\hline & 8 & Meliaceae & Cedrela odorata Linn. & 3 & 91.33 & 41.40 & 0.68 & 4.92 & -0.12 & 3.41 & 19.39 & 33.05 \\
\hline & 9 & Meliaceae & Trichilia heudelottii Planch. ex. Oliv. & 5 & 27.66 & 33.16 & 0.06 & 0.90 & -0.16 & 5.68 & 1.86 & 5.28 \\
\hline & 10 & Meliaceae & Trichilia prieuriana A. Juss & 3 & 25.60 & 32.37 & 0.07 & 0.64 & -0.12 & 3.41 & 1.95 & 3.32 \\
\hline & 11 & Moraceae & Trilepisium madagascariense Dc. Fl. Cam. & 5 & 25.24 & 24.48 & 0.06 & 0.50 & -0.16 & 5.68 & 1.84 & 5.22 \\
\hline & 12 & Olacaceae & Strobosia pustulata Oliv. & 3 & 14.10 & 17.97 & 0.02 & 0.10 & -0.12 & 3.41 & 0.46 & 0.78 \\
\hline & 13 & Rutaceae & Fagara leprieurii Engl. & 1 & 28.00 & 23.70 & 0.06 & 0.35 & -0.05 & 1.14 & 1.77 & 1.00 \\
\hline & 14 & Rutaceae & Fagara macrophylla (Oliv.) Engl & 1 & 35.80 & 26.10 & 0.10 & 0.66 & -0.05 & 1.14 & 2.89 & 1.64 \\
\hline & 15 & Sapotaceae & Chrysopyllum albidum G Don. & 1 & 33.00 & 35.30 & 0.09 & 1.07 & -0.05 & 1.14 & 2.46 & 1.40 \\
\hline & 16 & Sapotaceae & $\begin{array}{l}\text { Chrysopyllum perpulchrum Mildbr. ex } \\
\text { Hutch. \& Dalziel }\end{array}$ & 2 & 27.90 & 29.25 & 0.06 & 0.72 & -0.09 & 2.27 & 1.81 & 2.06 \\
\hline & 17 & Sterculiaceae & Cola gigantean A.Chev. & 4 & 41.10 & 32.63 & 0.22 & 2.62 & -0.14 & 4.55 & 6.24 & 14.17 \\
\hline & 18 & Sterculiaceae & Mansonia altissima A. Chev. & 10 & 36.57 & 30.00 & 0.13 & 1.02 & -0.25 & 11.36 & 3.60 & 20.47 \\
\hline & 19 & Sterculiaceae & Pterygota macrocarpa K Schum. & 4 & 31.38 & 32.15 & 0.10 & 1.12 & -0.14 & 4.55 & 2.99 & 6.79 \\
\hline & 20 & Sterculiaceae & Sterculia rhinopetala K. Schum. & 14 & 30.21 & 27.53 & 0.09 & 0.84 & -0.29 & 15.91 & 2.65 & 21.07 \\
\hline & 21 & Sterculiaceae & Triplochiton scleroxylon K. Schum. & 2 & 98.50 & 37.00 & 0.77 & 5.59 & -0.09 & 2.27 & 22.13 & 25.15 \\
\hline & 22 & Surmardaceae & Pierradendron africanum Hook . f & 1 & 43.10 & 58.00 & 0.15 & 2.06 & -0.05 & 1.14 & 4.19 & 2.38 \\
\hline & 23 & Ulmaceae & Celtis zenkeri Engl. & 1 & 15.40 & 14.00 & 0.02 & 0.14 & -0.05 & 1.14 & 0.53 & 0.30 \\
\hline & 24 & Verbaneaceae & Gmelina arborea Roxb. & 2 & 23.20 & 36.20 & 0.05 & 0.66 & -0.09 & 2.27 & 1.41 & 1.60 \\
\hline & & & Total & 88 & & & 3.48 & 30.02 & -2.82 & & & \\
\hline \multirow[t]{3}{*}{ PSP 29} & 1 & Annonaceae & Anogeissus leiocarpa (DC) Guill\&Perr. & 1 & 14.00 & 18.50 & 0.02 & 0.14 & -0.05 & 1.01 & 0.54 & 0.27 \\
\hline & 2 & Annonaceae & Cleistopholis patens (Benth.) Engl. \& Diels & 1 & 8.50 & 9.50 & 0.01 & 0.03 & -0.05 & 1.01 & 0.20 & 0.10 \\
\hline & 3 & Apocynaceae & Alstonia boonei De Wild. & 3 & 23.10 & 14.10 & 0.04 & 0.31 & -0.11 & 3.03 & 1.57 & 2.38 \\
\hline
\end{tabular}


Iyagin \& Adekunle (2017) 4(3): 496-513

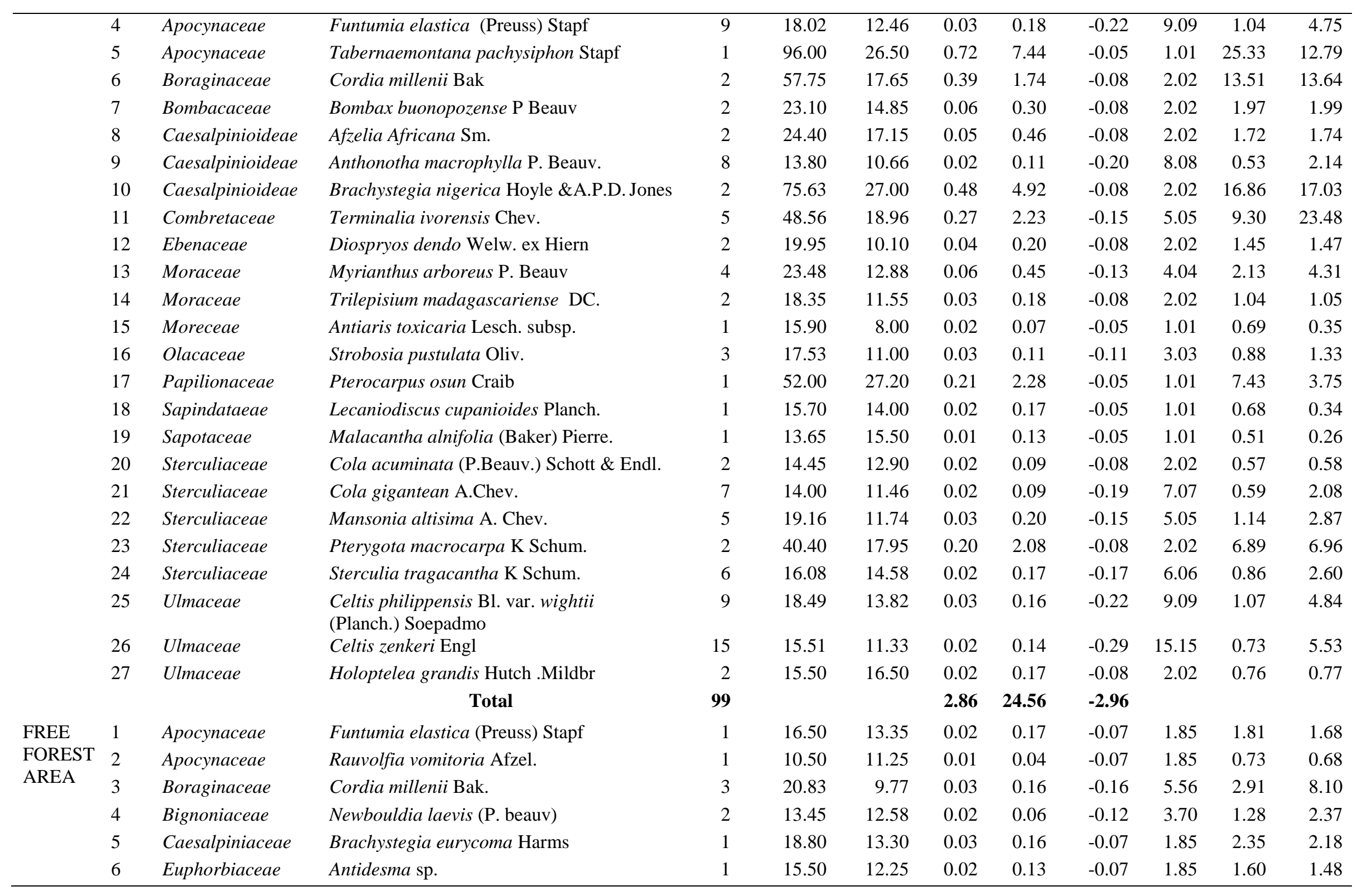

www.tropicalplantresearch.com 502 
Iyagin \& Adekunle (2017) 4(3): 496-513

\begin{tabular}{|c|c|c|c|c|c|c|c|c|c|c|c|c|}
\hline & 7 & Euphorbiaceae & Bridelia micrantha (Hochst.) Baill. & 5 & 20.26 & 10.99 & 0.04 & 0.18 & -0.22 & 9.26 & 3.04 & 14.10 \\
\hline & 8 & Euphorbiaceae & Macaranga barteri Müll.-Arg. & 1 & 9.40 & 13.40 & 0.01 & 0.04 & -0.07 & 1.85 & 0.59 & 0.54 \\
\hline & 9 & Meliaceae & Trichilia heldelotii Planch. ex. Oliv. & 2 & 16.75 & 11.38 & 0.02 & 0.13 & -0.12 & 3.70 & 1.90 & 3.52 \\
\hline & 10 & Mimoceae & Albizia adianthifolia (Schumach.) Wightii & 1 & 12.00 & 13.45 & 0.01 & 0.09 & -0.07 & 1.85 & 0.96 & 0.89 \\
\hline & 11 & Mimosaceae & Albizia zygia (DC.) J. F. Macbr. & 4 & 14.00 & 12.49 & 0.02 & 0.09 & -0.19 & 7.41 & 1.35 & 5.00 \\
\hline & 12 & Moraceae & Ficus exasperate Vahl & 1 & 12.70 & 12.55 & 0.01 & 0.08 & -0.07 & 1.85 & 1.07 & 0.99 \\
\hline & 13 & Moreaceae & Milicia excels (Welw.) C. Berg & 2 & 54.00 & 10.08 & 0.31 & 0.81 & -0.12 & 3.70 & 26.68 & 49.40 \\
\hline & 14 & Rutaceae & Zanthoxylum leprieurii Guill. \& Perr & 1 & 13.40 & 11.60 & 0.01 & 0.08 & -0.07 & 1.85 & 1.20 & 1.11 \\
\hline & 15 & Sapindaceae & Blighia sapida K Konig & 1 & 38.50 & 14.90 & 0.12 & 0.50 & -0.07 & 1.85 & 9.87 & 9.14 \\
\hline & 16 & Sterculiaceae & Cola gigantean A.Chev. & 1 & 10.00 & 11.50 & 0.01 & 0.07 & -0.07 & 1.85 & 0.67 & 0.62 \\
\hline & 17 & Sterculiaceae & Sterculia rhinopetela K. Schum. & 1 & 28.90 & 14.20 & 0.07 & 0.29 & -0.07 & 1.85 & 5.56 & 5.15 \\
\hline & 18 & Sterculiaceae & Sterculia tragacantha K Schum. & 1 & 11.50 & 6.78 & 0.01 & 0.05 & -0.07 & 1.85 & 0.88 & 0.82 \\
\hline & 19 & Sterculiaceae & Theobroma cacao L. & 1 & 12.00 & 5.90 & 0.01 & 0.04 & -0.07 & 1.85 & 0.96 & 0.89 \\
\hline & 20 & Sterculiaceae & Triplochiton scleroxylon K Schum. & 5 & 35.78 & 11.87 & 0.12 & 0.63 & -0.22 & 9.26 & 10.55 & 48.84 \\
\hline & 21 & Ulmaceae & Celtis philippensis $\mathrm{Bl}$. var. wightii & 5 & 14.30 & 10.00 & 0.02 & 0.08 & -0.22 & 9.26 & 1.37 & 6.36 \\
\hline & 22 & Ulmaceae & Celtis zenkeri Engl & 11 & 24.61 & 11.59 & 0.09 & 0.38 & -0.32 & 20.37 & 7.55 & 76.93 \\
\hline & 23 & Verbenaceae & Gmelina arborea Roxb & 2 & 47.25 & 13.75 & 0.18 & 0.69 & -0.12 & 3.70 & 15.09 & 27.95 \\
\hline & & & Total & 54 & & & 1.18 & 4.95 & -2.79 & & & \\
\hline \multirow[t]{13}{*}{ OLUWA } & 1 & Anacardiaceae & Lannea welwitschii (Hiern) Engl. & 2 & 19.00 & 13.05 & 0.03 & 0.31 & -0.05 & 1.18 & 0.81 & 0.47 \\
\hline & 2 & Annonaceae & Cleistopholis patens (Benth.) Engl. \& Diels & 1 & 17.20 & 11.50 & 0.02 & 0.25 & -0.03 & 0.59 & 0.64 & 0.19 \\
\hline & 3 & Annonaceae & Enantia chlorantha Oliv. & 1 & 22.50 & 9.50 & 0.04 & 0.27 & -0.03 & 0.59 & 1.10 & 0.32 \\
\hline & 4 & Annonaceae & Monodora myristica (Gaertn.) Dunal & 19 & 21.50 & 15.10 & 0.05 & 0.61 & -0.24 & 11.18 & 1.25 & 6.98 \\
\hline & 5 & Apocynaceae & Alstonia boonei De Wild. & 2 & 35.05 & 15.65 & 0.12 & 1.62 & -0.05 & 1.18 & 3.19 & 1.88 \\
\hline & 6 & Apocynaceae & Funtumia elastica (Preuss) Stapf & 4 & 24.10 & 17.35 & 0.05 & 0.46 & -0.09 & 2.35 & 1.49 & 1.76 \\
\hline & 7 & Apocynaceae & Hunteria umbellate K. Schum & 4 & 13.68 & 12.54 & 0.02 & 0.18 & -0.09 & 2.35 & 0.43 & 0.50 \\
\hline & 8 & Apocynaceae & $\begin{array}{l}\text { Picralima nitida (Stapf) T. Durand \& H. } \\
\text { Durand }\end{array}$ & 10 & 19.62 & 9.99 & 0.03 & 0.22 & -0.17 & 5.88 & 0.88 & 2.59 \\
\hline & 9 & Apocynaceae & Rauvolfia vomitoria Afzel. & 1 & 16.40 & 11.40 & 0.02 & 0.20 & -0.03 & 0.59 & 0.58 & 0.17 \\
\hline & 10 & Boraginaceae & Cordia millenii Bak. & 1 & 44.30 & 16.00 & 0.15 & 1.35 & -0.03 & 0.59 & 4.26 & 1.25 \\
\hline & 11 & Bignoniaceae & Newbouldia laevis (P. beauv) & 1 & 19.70 & 10.00 & 0.03 & 0.17 & -0.03 & 0.59 & 0.84 & 0.25 \\
\hline & 12 & Burseraceae. & Canarium schweinfurthii Engl. & 2 & 73.30 & 26.10 & 0.42 & 4.51 & -0.05 & 1.18 & 11.66 & 6.86 \\
\hline & 13 & Caesalpinioideae & Anthonatha macrophylla P. Beauv. & 3 & 12.03 & 10.90 & 0.01 & 0.09 & -0.07 & 1.76 & 0.32 & 0.28 \\
\hline
\end{tabular}

wWw.tropicalplantresearch.com 503 
Iyagin \& Adekunle (2017) 4(3): 496-513

\begin{tabular}{|c|c|c|c|c|c|c|c|c|c|c|c|}
\hline 14 & Capparaceae & Buchholzia coriacea Engl. & 13 & 29.28 & 16.90 & 0.12 & 2.60 & -0.20 & 7.65 & 3.23 & 12.34 \\
\hline 15 & Clusiaceae & Allanblackia floribunda Oliv. & 1 & 12.70 & 12.90 & 0.01 & 0.14 & -0.03 & 0.59 & 0.35 & 0.10 \\
\hline 16 & Clusiaceae & Garcinia afzeli Engl. & 2 & 19.75 & 10.25 & 0.03 & 0.22 & -0.05 & 1.18 & 0.87 & 0.51 \\
\hline 17 & Ebenaceae & Diospyros barteri Hiern. & 6 & 18.72 & 17.78 & 0.03 & 0.38 & -0.12 & 3.53 & 0.84 & 1.48 \\
\hline 18 & Ebenaceae & Diospyros dendo Welw. ex Hiern & 11 & 18.65 & 10.30 & 0.04 & 0.33 & -0.18 & 6.47 & 1.17 & 3.78 \\
\hline 19 & Ebenaceae & Diospyros mespiliformis Hochst. ex A.DC. & 6 & 21.10 & 11.24 & 0.04 & 0.44 & -0.12 & 3.53 & 1.14 & 2.01 \\
\hline 20 & Euphorbiaceae & Antidesma sp. & 2 & 11.40 & 10.95 & 0.01 & 0.08 & -0.05 & 1.18 & 0.28 & 0.17 \\
\hline 21 & Euphorbiaceae & Bridelia grandis Pierre ex. Hutch & 1 & 50.00 & 34.40 & 0.20 & 4.51 & -0.03 & 0.59 & 5.43 & 1.60 \\
\hline 22 & Euphorbiaceae & Croton sp. & 2 & 15.65 & 15.65 & 0.02 & 0.35 & -0.05 & 1.18 & 0.54 & 0.32 \\
\hline 23 & Euphorbiaceae & Ricinodendron heudelotii (Ball.) Pierre & 3 & 46.20 & 21.98 & 0.28 & 3.88 & -0.07 & 1.76 & 7.66 & 6.76 \\
\hline 24 & Lecythidaceae & Napoleonaea vogelii Hook. \& Planch. & 1 & 22.70 & 8.90 & 0.04 & 0.14 & -0.03 & 0.59 & 1.12 & 0.33 \\
\hline 25 & Meliaceae & Trichilia emetica Vahl & 1 & 13.50 & 14.22 & 0.01 & 0.74 & -0.03 & 0.59 & 0.40 & 0.12 \\
\hline 26 & Meliaceae & Trichilia heudelottii Planch. ex. Oliv. & 9 & 23.94 & 13.09 & 0.06 & 0.71 & -0.16 & 5.29 & 1.59 & 4.21 \\
\hline 27 & Meliaceae & Trichilia prieuriana A. Juss & 3 & 19.17 & 19.73 & 0.03 & 0.36 & -0.07 & 1.76 & 0.86 & 0.76 \\
\hline 28 & Mimosoideae & Albizia zygia (DC.) J. F. Macbr. & 1 & 14.70 & 15.90 & 0.02 & 0.27 & -0.03 & 0.59 & 0.47 & 0.14 \\
\hline 29 & Mimosoideae & Pentaclethra macrophylla Benth. & 1 & 11.11 & 9.60 & 0.01 & 0.05 & -0.03 & 0.59 & 0.27 & 0.08 \\
\hline 30 & Moraceae & $\begin{array}{l}\text { Antiaris toxicaria Lesch. subsp. } \\
\text { welwitschii (Engl.) C.C Berg }\end{array}$ & 2 & 44.30 & 20.00 & 0.16 & 1.06 & -0.05 & 1.18 & 4.30 & 2.53 \\
\hline 31 & Moraceae & Ficus exasperate Vahl & 2 & 23.50 & 17.45 & 0.05 & 0.46 & -0.05 & 1.18 & 1.29 & 0.76 \\
\hline 32 & Moraceae & Ficus sur Thunb. & 1 & 17.50 & 17.90 & 0.02 & 0.32 & -0.03 & 0.59 & 0.66 & 0.20 \\
\hline 33 & Moraceae & Milicia excels (Welw.) C. Berg & 1 & 10.70 & 18.80 & 0.01 & 0.08 & -0.03 & 0.59 & 0.25 & 0.07 \\
\hline 34 & Moraceae & Myrianthus arboreus P. Beauv. & 5 & 20.16 & 12.13 & 0.04 & 0.53 & -0.10 & 2.94 & 1.17 & 1.72 \\
\hline 35 & Moraceae & Trilepisium madagascariense Dc. Fl. Cam. & 1 & 37.30 & 8.00 & 0.11 & 0.39 & -0.03 & 0.59 & 3.02 & 0.89 \\
\hline 36 & Myristicaceae & Pycnanthus angolensis (Welw.) Warb. & 1 & 58.20 & 26.90 & 0.27 & 5.42 & -0.03 & 0.59 & 7.35 & 2.16 \\
\hline 37 & Myristicaceae & Staudtia stipitata Warb. & 1 & 24.60 & 14.40 & 0.05 & 0.42 & -0.03 & 0.59 & 1.31 & 0.39 \\
\hline 38 & Ochnaceae & Barteria fistulosa Mast. & 1 & 16.70 & 24.50 & 0.02 & 0.30 & -0.03 & 0.59 & 0.61 & 0.18 \\
\hline 39 & Olacaceae & Strobosia pustulata Oliv. & 2 & 36.95 & 16.30 & 0.16 & 2.17 & -0.05 & 1.18 & 4.31 & 2.54 \\
\hline 40 & Papilionoideae & Amphimas pterocarpoides Harms & 1 & 23.90 & 21.10 & 0.04 & 0.68 & -0.03 & 0.59 & 1.24 & 0.36 \\
\hline 41 & Papilionoideae & Baphia pubescens Hook. f. & 1 & 27.80 & 12.30 & 0.06 & 0.42 & -0.03 & 0.59 & 1.68 & 0.49 \\
\hline 42 & Papilionoideae & Dalbergia sp. & 1 & 35.80 & 32.00 & 0.10 & 1.78 & -0.03 & 0.59 & 2.78 & 0.82 \\
\hline 43 & Papilionoideae & Pterygota macrocarpa K Schum. & 2 & 16.75 & 29.70 & 0.02 & 0.72 & -0.05 & 1.18 & 0.66 & 0.39 \\
\hline 44 & Rubiaceae & Massularia acuminate & 1 & 12.20 & 7.20 & 0.01 & 0.04 & -0.03 & 0.59 & 0.32 & 0.10 \\
\hline
\end{tabular}


Iyagin \& Adekunle (2017) 4(3): 496-513

\begin{tabular}{|c|c|c|c|c|c|c|c|c|c|c|c|}
\hline 45 & Rubiaceae & Pausinystalia talbotii Wernham. & 2 & 29.35 & 15.65 & 0.07 & 0.80 & -0.05 & 1.18 & 1.92 & 1.13 \\
\hline 46 & Sapindaceae & Lecaniodis cuscupanioides Planch. & 1 & 26.40 & 23.50 & 0.05 & 1.17 & -0.03 & 0.59 & 1.51 & 0.44 \\
\hline 47 & Sapotaceae & Chrysophyllum albidum G Don. & 1 & 47.50 & 23.70 & 0.18 & 2.20 & -0.03 & 0.59 & 4.90 & 1.44 \\
\hline 48 & Sapotaceae & Malacantha alnifolia (Baker) Pierre. & 3 & 14.53 & 12.03 & 0.02 & 0.19 & -0.07 & 1.76 & 0.47 & 0.41 \\
\hline 49 & Sterculiaceae & Sterculiar hinopetala K. Schum. & 6 & 23.58 & 14.27 & 0.05 & 0.49 & -0.12 & 3.53 & 1.34 & 2.37 \\
\hline 50 & Sterculiaceae & Cola gigantea A.Chev. & 1 & 17.90 & 13.00 & 0.03 & 0.23 & -0.03 & 0.59 & 0.70 & 0.20 \\
\hline 51 & Sterculiaceae & Cola heterophylla (P.Beauv.) Schott \& Endl & 5 & 18.84 & 15.05 & 0.03 & 0.43 & -0.10 & 2.94 & 0.93 & 1.37 \\
\hline 52 & Sterculiaceae & Cola nigerica Brenan \& Keay & 2 & 13.80 & 8.65 & 0.01 & 0.06 & -0.05 & 1.18 & 0.41 & 0.24 \\
\hline 53 & Sterculiaceae & Sterculia tragacantha K Schum. & 4 & 12.50 & 12.80 & 0.01 & 0.09 & -0.09 & 2.35 & 0.34 & 0.40 \\
\hline 54 & Ulmaceae & Celtis zenkeri Engl & 8 & 29.25 & 17.78 & 0.10 & 1.05 & -0.14 & 4.71 & 2.87 & 6.75 \\
\hline & & Total & 170 & - & - & 3.62 & 46.95 & -3.55 & - & - & - \\
\hline
\end{tabular}

Note: nha $^{-1}$ - number of stem per hectare, MDBH - Mean Diametre at breast height $(\mathrm{cm}), \mathrm{M}$ Ht -Mean height $(\mathrm{m}), \mathrm{BA}-\mathrm{Basal}$ area per hectare $\left(\mathrm{m}^{2}\right), \mathrm{VOL}-\mathrm{Volume}$ per hectare $\left(\mathrm{m}^{3}\right), \mathrm{RD}$ -

Spp. Relative density, $\mathrm{RD}_{\mathrm{O}}$ - Spp. Relative dominance. 
Table 2. Families' importance index for the study areas.

\begin{tabular}{|c|c|c|c|c|c|c|c|}
\hline S.N. & Families & BA/ha & VOL/ha & $\mathbf{R F}$ & $\mathrm{RD}(\%)$ & RDO (\%) & FIV \% \\
\hline 1 & Anacardiaceae & 0.03 & 0.31 & 0.49 & 0.49 & 0.01 & 0.98 \\
\hline 2 & Annonaceae & 0.10 & 1.01 & 6.33 & 6.33 & 0.03 & 12.71 \\
\hline 3 & Apocynaceae & 0.21 & 1.68 & 11.92 & 11.92 & 0.05 & 23.95 \\
\hline 4 & Bignoniaceae & 0.50 & 2.62 & 2.19 & 2.19 & 0.13 & 4.52 \\
\hline 5 & Bombacaceae & 0.06 & 0.30 & 0.49 & 0.49 & 0.01 & 0.99 \\
\hline 6 & Burseraceae & 0.31 & 2.71 & 1.22 & 1.22 & 0.08 & 2.52 \\
\hline 7 & Caesalpinioideae & 0.14 & 1.22 & 3.89 & 3.89 & 0.03 & 7.84 \\
\hline 8 & Capparaceae & 0.12 & 2.60 & 3.16 & 3.16 & 0.03 & 6.37 \\
\hline 9 & Clusiaceae & 0.03 & 0.24 & 0.49 & 0.49 & 0.01 & 0.98 \\
\hline 10 & Combretaceae & 0.27 & 2.23 & 1.22 & 1.22 & 0.07 & 2.51 \\
\hline 11 & Ebenaceae & 0.12 & 0.86 & 6.33 & 6.33 & 0.03 & 12.71 \\
\hline 12 & Euphorbiaceae & 0.19 & 2.50 & 3.89 & 3.89 & 0.05 & 7.85 \\
\hline 13 & Guttiferae & 0.02 & 0.10 & 0.24 & 0.24 & 0.01 & 0.49 \\
\hline 14 & Lecythidaceae & 0.29 & 2.07 & 0.97 & 0.97 & 0.07 & 2.02 \\
\hline 15 & Malvaceae & 0.03 & 0.18 & 0.73 & 0.73 & 0.01 & 1.47 \\
\hline 16 & Meliaceae & 0.30 & 2.68 & 6.33 & 6.33 & 0.08 & 12.76 \\
\hline 17 & Mimosoideae & 0.03 & 0.25 & 1.70 & 1.70 & 0.01 & 3.42 \\
\hline 18 & Moraceae & 0.39 & 1.92 & 6.57 & 6.57 & 0.10 & 13.27 \\
\hline 19 & Myristicaceae & 0.16 & 2.92 & 0.49 & 0.49 & 0.04 & 1.01 \\
\hline 20 & Ochnaceae & 0.02 & 0.30 & 0.24 & 0.24 & 0.01 & 0.49 \\
\hline 21 & Olacaceae & 0.20 & 2.38 & 1.95 & 1.95 & 0.05 & 3.95 \\
\hline 22 & Papilionoideae & 0.26 & 3.14 & 1.46 & 1.46 & 0.07 & 2.99 \\
\hline 23 & Rubiaceae & 0.05 & 0.55 & 0.73 & 0.73 & 0.01 & 1.48 \\
\hline 24 & Rutaceae & 0.10 & 0.59 & 0.73 & 0.73 & 0.02 & 1.49 \\
\hline 25 & Sapindaceae & 0.19 & 1.84 & 0.73 & 0.73 & 0.05 & 1.51 \\
\hline 26 & Sapotaceae & 0.13 & 1.45 & 1.95 & 1.95 & 0.03 & 3.94 \\
\hline 27 & Sterculiaceae & 0.32 & 2.55 & 19.46 & 19.46 & 0.08 & 39.10 \\
\hline 28 & Surmardaceae & 0.15 & 2.06 & 0.24 & 0.24 & 0.04 & 0.52 \\
\hline 29 & Ulmaceae & 0.21 & 1.62 & 12.41 & 12.41 & 0.05 & 24.93 \\
\hline \multirow[t]{2}{*}{30} & Verbenaceae & 0.23 & 1.34 & 0.97 & 0.97 & 0.06 & 2.01 \\
\hline & Total & 5.13 & 46.23 & & & & \\
\hline
\end{tabular}

Biodiversity Indices

The summary of the abundance, level of diversity and evenness of all the tree species encountered with the various biodiversity indices is presented in table 3. These indices were used to compare tree species diversity of the selected forest communities. Oluwa Forest Reserve has the highest Shannon-Weiner index (3.55), indicating that it has the highest species richness when compared with the other locations. The Pielou's evenness (equitability index) and Margalef's index value of 0.69 and 10.12 respectively were also obtained for this forest reserve and 34.74 and 11.11 were obtained respectively for number 1 and number 2 of Hill's diversity indices. The free forest area adjoining PSP 29 has the lowest Shannon-wiener diversity index (2.79), Margalef's index value of 5.51 but, the highest Pielou's evenness index value of 0.70 .

Table 3. Summary of the tree species diversity indices in the selected areas.

\begin{tabular}{lrrrrr}
\hline \multirow{2}{*}{ Variables } & \multicolumn{5}{c}{ Locations } \\
\cline { 2 - 6 } & SNR & PSP (29) & Free Are & $\begin{array}{r}\text { Oluwa } \\
\text { FR }\end{array}$ & $\begin{array}{r}\text { All } \\
\text { locations }\end{array}$ \\
\hline No of trees/ ha & 88 & 99 & 54 & 170 & 411 \\
No of Families (NF) & 15 & 14 & 12 & 23 & 30 \\
No of Species (NS) & 24 & 27 & 23 & 53 & 78 \\
Shannon- Wieners (H ${ }^{1}$ ) & 2.82 & 2.96 & 2.79 & 3.55 & 5.11 \\
Pielou's spp evenness index (E) & 0.63 & 0.64 & 0.70 & 0.69 & 0.69 \\
Simpson's concentration ( $\lambda$ ) & 0.07 & 0.07 & 0.18 & 0.09 & 0.03 \\
N1 of hill diversity (N1) & 16.81 & 19.29 & 16.23 & 34.74 & 35.40 \\
N2 of hill diversity (N2) & 14.28 & 14.28 & 5.55 & 11.11 & 33.33 \\
Margalef's index of Spp. richness (M) & 5.13 & 5.65 & 5.51 & 10.12 & 12.79 \\
\hline
\end{tabular}

Tree growth variables in the selected forest reserves

The summary of the tree growth variables according to the location is shown in table 4. Oluwa forest 
Table 4. Tree growth variables for the selected area.

\begin{tabular}{lrrrrr}
\hline Variables & SNR & PSP 29 & Free Art & $\begin{array}{r}\text { Oluwa } \\
\text { FR }\end{array}$ & All locations \\
\hline Basal area per ha $\left(\mathrm{m}^{2}\right)$ & 12.31 & 6.73 & 3.40 & 10.54 & 48.48 \\
Volume per ha $\left(\mathrm{m}^{3}\right)$ & 104.76 & 53.44 & 14.56 & 141.06 & 123.10 \\
Mean DBH $(\mathrm{cm})$ & 35.15 & 27.15 & 20.48 & 24.57 & 25.06 \\
Dominant DBH $(\mathrm{cm})$ & 98.50 & 96.00 & 54.00 & 73.30 & 98.50 \\
Dominant height $(\mathrm{m})$ & 58.00 & 27.20 & 14.90 & 34.40 & 58.00 \\
Mean height (m) & 28.1 & 14.92 & 12.09 & 15.87 & 17.09 \\
\hline
\end{tabular}

reserve has the highest tree volume per hectare of $141.06 \mathrm{~m}^{3}$ while the free Forest has the lowest tree volume per hectare $\left(14.56 \mathrm{~m}^{3}\right)$. The highest dominant DBH is $98.50 \mathrm{~cm}$ in the SNR, followed by PSP $29(96.00 \mathrm{~cm})$ while the lowest dominant DBH of $54 \mathrm{~cm}$ was obtained in the free forest area. The highest mean DBH value of 35.15 $\mathrm{cm}$ was also recorded in the SNR. The basal area/ha ranged between $3.40 \mathrm{~m}^{2}$ and $12.31 \mathrm{~m}^{2}$, with the least obtained at the disturbed free area and the highest at the SNR. Similarly, the dominant height and Mean height followed the same trend. The dominant height value ranged between $14.90 \mathrm{~m}$ and $58.00 \mathrm{~m}$ while the value for mean height ranged from 12.09 to 28.1 respectively.

\section{Diameter and height Distribution}

Table 5. Species diversity, abundance, basal area and volume distribution of the study area into diameter classes.

\begin{tabular}{|c|c|c|c|c|c|c|}
\hline Location & DBH Class (cm) & NS & NF & $\mathbf{N i}$ & Ba. $h^{-1}\left(\mathbf{m}^{2}\right)$ & Vol.h ${ }^{-1}\left(\mathrm{~m}^{3}\right)$ \\
\hline \multirow[t]{7}{*}{ SNR } & $0-20$ & 17 & 11 & 34 & 0.61 & 5.47 \\
\hline & $21-40$ & 15 & 10 & 27 & 1.81 & 17.68 \\
\hline & $41-60$ & 7 & 5 & 13 & 2.45 & 21.06 \\
\hline & $61-80$ & 5 & 4 & 7 & 2.47 & 21.79 \\
\hline & $81-100$ & 5 & 4 & 5 & 2.92 & 24.84 \\
\hline & $>100$ & 2 & 2 & 2 & 1.95 & 13.92 \\
\hline & Total & 24 & 15 & 88 & 12.21 & 104.76 \\
\hline \multirow[t]{7}{*}{ Oluwa Forest Reserve } & $0-20$ & 38 & 21 & 94 & 1.53 & 14.43 \\
\hline & $21-40$ & 29 & 17 & 60 & 3.80 & 43.80 \\
\hline & $41-60$ & 9 & 9 & 10 & 1.92 & 28.38 \\
\hline & $61-80$ & 3 & 3 & 3 & 1.01 & 11.09 \\
\hline & $81-100$ & 2 & 2 & 2 & 1.29 & 15.27 \\
\hline & $>100$ & 1 & 1 & 1 & 0.99 & 28.09 \\
\hline & Total & 50 & 23 & 170 & 10.54 & 141.06 \\
\hline \multirow[t]{6}{*}{ PSP 29} & $0-20$ & 24 & 13 & 67 & 1.00 & 6.05 \\
\hline & $21-40$ & 13 & 8 & 22 & 1.28 & 8.21 \\
\hline & $41-60$ & 3 & 3 & 3 & 0.57 & 5.23 \\
\hline & $61-80$ & 3 & 3 & 3 & 1.01 & 8.71 \\
\hline & $81-100$ & 4 & 4 & 4 & 2.87 & 25.24 \\
\hline & Total & 27 & 14 & 99 & 6.73 & 53.44 \\
\hline \multirow[t]{5}{*}{ Free Forest Area } & $0-20$ & 19 & 10 & 40 & 0.69 & 3.93 \\
\hline & $21-40$ & 7 & 6 & 7 & 0.45 & 1.96 \\
\hline & $41-60$ & 4 & 3 & 5 & 1.03 & 5.00 \\
\hline & $61-80$ & 2 & 2 & 2 & 1.23 & 3.67 \\
\hline & Total & 23 & 12 & 54 & 3.40 & 14.56 \\
\hline
\end{tabular}

Note: NF - Number of Family, NS - Number of species, Ni - Number of individual species.

The diameter distribution of the study areas is presented in tables 5 . This shows that $49.4 \%$ of trees falls within the lowest diameter class $(10-20 \mathrm{~cm})$ while only $7.14 \%$ falls in the highest diameter class $(>80 \mathrm{~cm})$. The highest Volume per hectare $\left(141.06 \mathrm{~m}^{3}\right)$ was recorded in Oluwa forest while the lowest $\left(14.56 \mathrm{~m}^{3}\right)$ was from the free Forest. The same trend was noted in the distribution of the individual species and families into diameter classes. Figure 2 was used to describe the tree species population structure based on DBH (cm) classes with frequency and volume per hectare $\left(\mathrm{m}^{3}\right)$. As one of the peculiar features of a mature natural forest ecosystem, the DBH distribution curve followed inverse J-shaped pattern (Fig. 3). Table 6 revealed that $49.4 \%$ of the tree species was in the height class of between 11-20 m in Oluwa Forest reserve. Only the SNR and Oluwa forest reserve have trees with height above $30 \mathrm{~m}$ (18.7\% and $7.02 \%$ respectively). These are trees that can be regarded as emergent. Significant differences $(\mathrm{P}$ - value $<0.05)$ exist in all the tree growth variables of the study sites when compared with one-way ANOVA (Table 7). Table 8 revealed the results of the mean separation of tree 
growth variables of the forests with Duncan Multiple Range Test (DMRT). The Mean values in the same row followed by the same letter(s) are not significantly different at 0.05 level of significance.

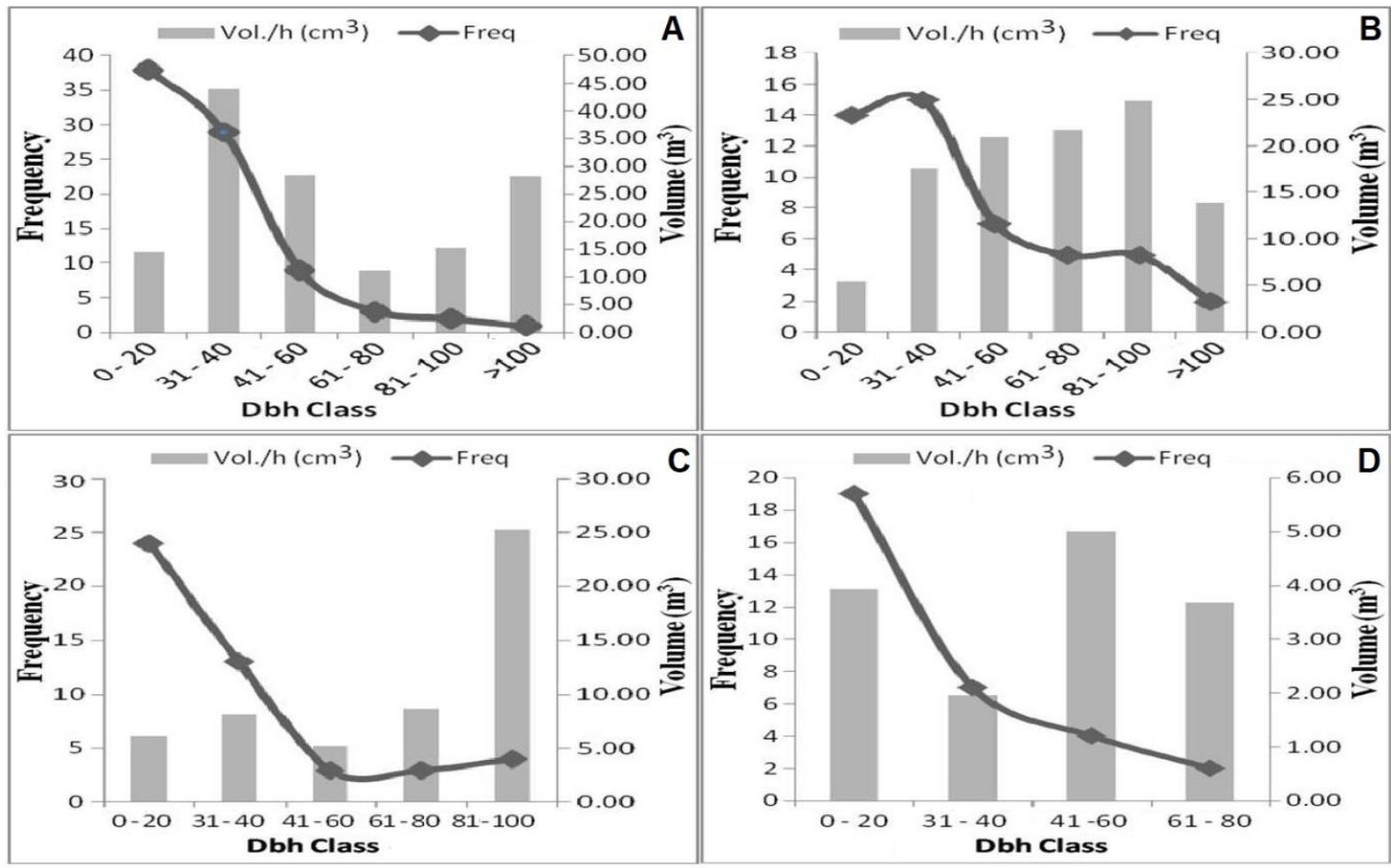

Figure 2. Tree species population structure of the study areas based on DBH $(\mathrm{cm})$ classes with frequency and volume $\left(\mathrm{m}^{3}\right)$ (A, Oluwa Forest Reserve; B, SNR Aponmu; C, PSP 29; D, Free Forest).

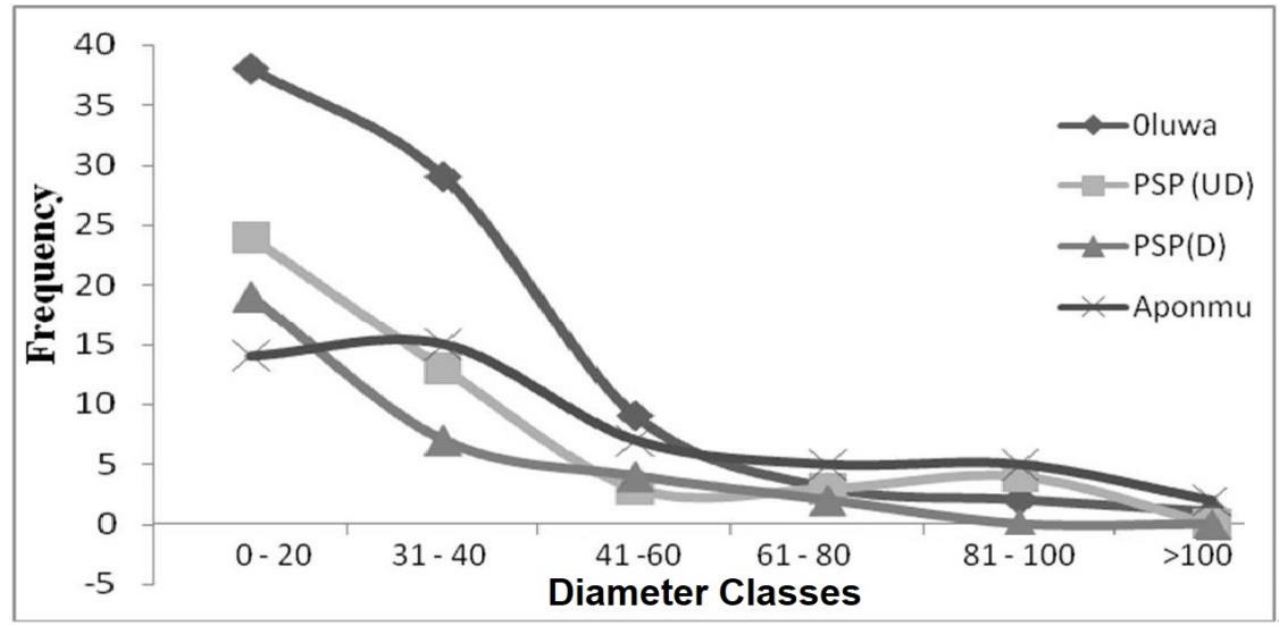

Figure 3. Diameter distribution curve for the selected Protected Areas and the free area.

Table 6. Species diversity, basal area and volume distribution of the study area into Height classes.

\begin{tabular}{|c|c|c|c|c|c|c|}
\hline & Height (m) & NS & NF & $\mathbf{N i}$ & Ba.h ${ }^{-1}\left(\mathrm{~cm}^{2}\right)$ & Vol.h $^{-1}\left(\mathrm{~cm}^{3}\right)$ \\
\hline \multirow[t]{6}{*}{ SNR } & $<10$ & 1 & 1 & 1 & 0.03 & 0.06 \\
\hline & $11-20$ & 14 & 10 & 24 & 1.08 & 7.21 \\
\hline & $21-30$ & 13 & 9 & 28 & 1.9 & 14.65 \\
\hline & $31-40$ & 12 & 6 & 20 & 4.62 & 38.54 \\
\hline & $41-50$ & 12 & 6 & 15 & 4.59 & 44.3 \\
\hline & Total & & & 88 & 12.22 & 104.76 \\
\hline \multirow[t]{6}{*}{ Oluwa } & $<10$ & 23 & 19 & 47 & 1.17 & 5.34 \\
\hline & $11-20$ & 39 & 20 & 92 & 4.01 & 41.27 \\
\hline & $21-30$ & 16 & 15 & 21 & 3.37 & 45.83 \\
\hline & $31-40$ & 7 & 7 & 8 & 0.96 & 19.2 \\
\hline & $41-50$ & 2 & 1 & 2 & 1.02 & 29.41 \\
\hline & Total & & & 170 & 10.53 & 141.05 \\
\hline
\end{tabular}


Iyagin \& Adekunle (2017) 4(3): 496-513

\begin{tabular}{llrrrrr}
\hline PSP 29 & \multicolumn{10}{l}{} & 18 & 10 & 32 & 0.4 & 1.87 \\
& $11-20$ & 18 & 12 & 52 & 2.16 & 12.36 \\
& $21-30$ & 12 & 8 & 15 & 4.18 & 39.22 \\
& Total & & & $\mathbf{9 9}$ & $\mathbf{6 . 7 4}$ & $\mathbf{5 3 . 4 5}$ \\
\hline Free & $<10$ & 8 & 5 & 13 & 1.51 & 4.63 \\
Forest & $11-20$ & 22 & 11 & 41 & 1.9 & 9.95 \\
area & Total & & & $\mathbf{5 4}$ & $\mathbf{3 . 4 1}$ & $\mathbf{1 4 . 5 8}$ \\
\hline
\end{tabular}

Table 7. ANOVA table for comparing differences in tree growth variables among the study sites.

\begin{tabular}{|c|c|c|c|c|c|c|}
\hline & $\begin{array}{l}\text { Source of } \\
\text { variation }\end{array}$ & SS & Df & MS & $\mathbf{F}$ & Sig \\
\hline \multirow[t]{3}{*}{ Vol. } & Location & 2418.06 & 3 & 806.02 & 5.70 & 0.01 \\
\hline & Error & 1695.62 & 12 & 141.30 & & \\
\hline & Total & 4113.69 & 15 & & & \\
\hline \multirow[t]{3}{*}{$\mathbf{B A}$} & Location & 11.99 & 3 & 4.00 & 3.76 & 0.04 \\
\hline & Error & 12.76 & 12 & 1.06 & & \\
\hline & Total & 24.75 & 15 & & & \\
\hline \multirow[t]{3}{*}{ DBH } & Location & 1090061.90 & 3 & 363353.97 & 9.14 & 0.00 \\
\hline & Error & 476814.23 & 12 & 39734.52 & & \\
\hline & Total & 1566876.14 & 15 & & & \\
\hline \multirow[t]{3}{*}{$\mathbf{H t}$} & Location & 679483.31 & 3 & 226494.44 & 22.75 & 0.00 \\
\hline & Error & 119471.91 & 12 & 9955.99 & & \\
\hline & Total & 798955.22 & 15 & & & \\
\hline \multirow[t]{3}{*}{ Nha } & Location & 1782.69 & 3 & 594.23 & 17.45 & 0.00 \\
\hline & Error & 408.75 & 12 & 34.06 & & \\
\hline & Total & 2191.44 & 15 & & & \\
\hline
\end{tabular}

Table 8. Comparison of growth variables in the forests with Duncan multiple range test (DMRT).

\begin{tabular}{lcccc}
\hline Properties & SNR & Oluwa & Free Forest & PSP 29 \\
\hline Volume & $26.19 \pm 11.62 \mathrm{ab}$ & $35.96 \pm 20.02 \mathrm{a}$ & $3.64 \pm 0.83 \mathrm{c}$ & $13.36 \pm 5.34 \mathrm{bc}$ \\
Basal Area & $3.06 \pm 1.31 \mathrm{a}$ & $2.68+ \pm 1.34 \mathrm{a}$ & $0.85 \pm 0.17 \mathrm{~b}$ & $1.68 \pm 0.81 \mathrm{ab}$ \\
DBH & $759.97 \pm 227.62 \mathrm{ab}$ & $1012.22 \pm 295.55 \mathrm{a}$ & $303.37 \pm 51.98 \mathrm{c}$ & $553.67 \pm 130.64 \mathrm{bc}$ \\
Height & $625.56 \pm 145.23 \mathrm{a}$ & $649.13 \pm 121.70 \mathrm{a}$ & $155.25 \pm 51.95 \mathrm{c}$ & $336.90 \pm 34.93 \mathrm{~b}$ \\
N/ha & $22.0 \pm 3.91 \mathrm{~b}$ & $42.50 \pm 8.38 \mathrm{a}$ & $13.50 \pm 4.79 \mathrm{c}$ & $24.75 \pm 5.25 \mathrm{bc}$ \\
\hline
\end{tabular}

\section{DISCUSSION}

There are two different conservation methods of natural resources in Nigeria; the in-situ and ex situ. Different types of PAs aimed at conserving the natural resources in their natural habitat, such as those used in this study, are examples of an in-situ conservation method. The tropical rainforest ecosystem of southwest Nigeria is noted for its high diversity in terms of species, genetic materials and ecological processes in comparison to other ecosystems (Adekunle et al. 2013). This assertion is supported by the results of this present study where 78 species in 30 families were encountered selected PAs. When these different forest communities were compared, the undisturbed PAs (PSP 29; SNR, and Oluwa Forest Reserve) were more diverse than the free forest area that has witnessed human activities. The low number of stems.ha ${ }^{-1}$ recorded in the free area, compared to the PAs, is an indication of an ecosystem that has been disturbed and greatly degraded. The number of species encountered in the sample survey plots can be used as a substitute for the exact species richness in the study area. Though, varying degree of degradation through illegal logging, forest conversion to Agricultural uses among others has resulted to unsustainable management of the lowland humid forest. The tropical forests are very vulnerabile to deforestation and degradation (FAO 2001, FAO 2006, Onyekwelu et al. 2005, Lafrankie et al. 2006). As a result, there is reduction in tree species richness, abundance and evenness of the ecosystem (Lafrankie et al. 2006) which is not creditable to biodiversity conservation. The tree species distribution into families agrees with the work of Adekunle et al. (2010) who indicated that the tropical rainforest ecosystem of southwest Nigeria is dominated by some specific families such as the Sterculiaceae, Meliaceae, Moraceae and Ebeneceae. Isichei (1995) also reported that Nigerian rainforest ecosystem is dominated by members of Sterculiaceae, Moraceae, Ulmaceae and Meliaceae families. The dominant families represented in this study sites differ from the families (Euphorbiaceae, Mimosoideae, Rubiaceae and Guttiferae) reported by Ifo et al. (2016) in their study of a similar ecosystem.

www.tropicalplantresearch.com 
This study revealed a decrease in species richness and diversity from the undisturbed forests to the disturbed forest. The number of trees per hectare (170, 99, 88 and 54 for Oluwa forest, PSP 29, SNR, and the free forest area, respectively) are similar to the number of tree per hectare recorded for the tropical natural forest by Parthasarathy (2001) but was far below the values obtained for Kalapahad $\left(579 \mathrm{ha}^{-1}\right)$ and Macarthy Valley (732 $\mathrm{ha}^{-1}$ ) in the giant evergreen forest of Andaman and Nicobar Islands as reported by Rajkumar \& Parthasarathy (2008). The highest number of tree species (50 species from 23 families) was recorded in Oluwa Forest (undisturbed), indicating richness in species while the lowest number of species ( 23 species from 12 families) was recorded in the free forest site (disturbed site). This reveals a lower level of species richness (Table 5). Simillar trend as this was reported by Borah et al. (2014). This is due to more frequent and heavy extraction of forest resources such as collection of fuel wood and NTFPs by the Local people. The number of family (30), number of Species (78) and Species Evenness (0.69) in this study corresponds to the results of Adekunle \& Olagoke (2008) as well as Onyekwelu et al. (2007) but lesser when compared to the studies of Lu et al. (2010), Rajkumar \& Parthasarathy (2008). These researchers reported a total of 95 species and 105 species in Xishuangbana, Chain tropical rainforest and India evergreen forest of Andaman Giant respectively. Various researchers have considered the use of Shannon wiener diversity index for the determination of forest community diversity in the tropics (Onyekwelu et al. 2007, Adekunle \& Olagoke 2008, Borah et al. 2014, Boboye \& Jimoh 2016). Shannon Diversity Index values for this study ranged between 2.79 and 3.55 . These values fall within the range (between 0.70 and 3.57) reported for tropical forests (Bhuyan et al. 2003, Bajpai et al. 2012, Borah et al. 2014, Sarkar \& Devi 2014, Bajpai et al. 2015, Vinayaka \& Krishnamurthy 2016). However, the overall Shannon-Weiner diversity index for the whole study areas (5.11) is higher than what was obtained by Onyekwelu et al. (2007) and Kent \& Coker (1992). The Pielou's species evenness of the entire study areas (0.69) is higher than 0.66 and 0.55 reported by Onyekwelu et al. (2005) and Adekunle (2006) respectively but less than the 0.82 that was recorded by Adekunle et al. (2013) when he examined the efficacy of Strict Nature Reserves (SNR) as a means of biodiversity conservation. This finding indicated that forest exploitation could lead to a drastic reduction in species diversity but might increase stand density in terms of number of tree stems per hectare.

As one of the peculiar features of a mature natural forest ecosystem, the DBH distribution curve of tree species followed inverse J-shaped pattern (Figs. 2 \& 3). The same was reflected in the study of Adekunle et al. 2013. Highest proportion of the trees were within the lowest diameter class $(10-20 \mathrm{~cm})$ and the least percentage in the highest diameter class (>80cm). In comparison, Huang et al. (2003) and Lu et al. (2010) reported a lesser percentage (4.5\% and $3.5 \%$ ) for trees that falls within the highest diameter class in forests of similar ecosystem as the study area.

Similarly, the same trend was noticed in the distribution of the individual species and families into diameter classes. Oluwa forest recorded the highest Vol.ha ${ }^{-1}\left(141.06 \mathrm{~m}^{3}\right)$ and the lowest value $\left(14.56 \mathrm{~m}^{3}\right)$ was from the free forest area adjoining Permanent Sample Plot 29, representing a degraded forest. Only the SNR and Oluwa forest reserve have trees with height above $30 \mathrm{~m}$ (18.7\% and $7.02 \%$ respectively). These are trees that can be regarded as emergent. Going by the recommended value of $25 \mathrm{~m}^{2}$.ha ${ }^{-1}$ for mean basal area of a well-stocked forest (Alder \& Abayomi 1994), the mean basal areas obtained in some of the forest reserves in the study areas shows that the forest reserves are not yet well stocked. The variation in the basal area of the forests (ranged from 3.40 to $12.31 \mathrm{~m}^{2}$.ha ${ }^{-1}$ ) is significantly lower to the recommended basal area.

\section{CONCLUSION AND RECOMMENDATION}

The results of this study revealed the present phytosociological properties of selected PAs in the humid forest ecosystem in terms of tree species diversity, evenness, and stand bio-volume. The study also demonstrated the importance of in-situ conservation method and the detrimental effect of forest degradations to tree species diversity and abundance. The biodiversity indices were high and the forest structure shows a continuous growth feature until they reach maturity and a stable state. Therefore, logging, hunting, farming and human settlement should stop in the free forest areas and be delineated as PAs while the existing in-situ conserved areas should be strictly monitored against any form of anthropogenic activities.

For greater production, conservation and sustainability of the protected areas, the government and nongovernmental organizations should provide farmers with incentives as motivation tool to enhance the forest and its resources. More attention should therefore be paid to species with narrow range and those threatened with extinction in the ecosystem through the conservation of their genetic material such as seed ex-situ conservation. 


\section{ACKNOWLEDGEMENTS}

We wish to appreciate the Director of Forestry in Ondo State and the Forestry Research Institute of Nigeria (FRIN) for giving us permit to access the Protected Areas within their Jurisdictions where data was collected for this work.

\section{REFERENCE}

Adeduntan SA \& Olusola JA (2013) Diversity and Abundance of Arthropods and Tree Species as Influenced by Different Forest Vegetation types in Ondo State, Nigeria. International Journal of Ecosystem 3(3): 19-23.

Adeduntan SA (2009) Diversity and abundance of soil mesofauna and microbial population in South-Western Nigeria. African Journal of Plant Science 3(9): 210-216.

Adekunle VAJ \& Dafiwhare HB (2011) Diversity and abundance of microbes, pH and organic matter in soils of different forest types in tropical humid lowland forest ecosystem, Nigeria. Journal of Biodiversity and Ecological Sciences 1(4): 333-342.

Adekunle VAJ \& Fatade T (2007) Trade and utilization of useful Medicinal Plants from Tropical Forest Ecosystem in Ondo State. In: Onibi GE, Agele SO, Adekunle VAJ \& Akinbulumo MO (eds) Medicinal plants in agriculture: the Nigerian experience. Proceedings of the third Annual Conference of the School of Agriculture and Agricultural Technology, Federal University of Technology, Akure, Nigeria/ Humboldt Kellong held on $14^{\text {th }}$ to $19^{\text {th }}$ April, 2007. pp. 291-297.

Adekunle VAJ \& Olagoke AO (2008) Diversity and biovolume of tree species in natural forest ecosystem in the bitumen-producing area of Ondo State, Nigeria.ll A baseline study. Biodiversity and Conservation 17: 27352755.

Adekunle VAJ (2006) Conservation of tree species diversity in tropical rainforest ecosystem of south-west Nigeria. Journal of Tropical Forest Science 18(2): 91-101.

Adekunle VAJ, Nair NK, Srivastava AK \& Singh NK (2014) Volume yield, tree species diversity and carbon hoard in protected areas of two developing countries. Forest Science \& Technology 10(2): 89-103.

Adekunle VAJ, Olagoke AO \& Akindele SO (2013) Tree species diversity and structure of a Nigerian strict nature reserve. Tropical Ecology 54(3): 275-289.

Adekunle VAJ, Olagoke AO \& Ogundare LF (2010) Rate of timber production in a tropical rainforest ecosystem of southwest Nigeria and its implications on sustainable forest management. Journal of Forestry Research 21: 225-230.

Aidar MP, Godoy JR, Bergmann J \& Joly CA (2001) “Atlantic Forest succession over calcareous soil, Parque Estadual Tur' 1stico do Alto Ribeira_PETAR, SP,” Revista Brasileira de Bot ^anica 24 (4): 455-469.

Aju PC \& Ezeibekwe IO (2010) Understanding and appreciating the need for biodiversity conservation in Nigeria. Journal of Medicinal Plants Research 4 (24): 2605-2608.

Alder D \& Abayomi JO (1994) Assessment of data requirement for sustained yield Calculations. A Consultancy Report Prepared for the Nigerian Tropical Action Plan.

Bajpai O, Kumar A, Mishra AK, Sahu N, Pandey J, Behera SK \& Chaudhary LB (2012) Recongregation of tree species of Katerniaghat wildlife sanctuary, Uttar Pradesh, India. Journal of Biodiversity \& Environmental Sciences 2: 24-40.

Bajpai O, Kushwaha AK, Srivastava AK, Pandey J \& Chaudhary LB (2015) Phytosociological status of a monotypic genus Indopiptadenia: A near threatened tree from the Terai-Bhabar region of central Himalaya. Research Journal of Forestry 9: 35-47.

Behera SK, Mishra AK, Sahu N, Kumar A, Singh N, Kumar A, Bajpai O, Chaudhary LB, Khare PB \& Tuli R (2012) The study of microclimate in response to different plant community association in tropical moist deciduous forest from northern India. Biodiversity and Conservation 21(5): 1159-1176.

Bhuyan P, Khan ML \& Tripathi RS (2003) The Tree diversity and population structure in undisturbed and human impacted stands of tropical wet evergreen forest in Arunachal Pradesh, Eastern Himalayas, India. Biodiversity Conservation 12: 1753-1773.

Boboye O \& Jimoh SO (2016) Pattern of plant species diversity in a dry Forest ecosystem of Nigeria. Journal of Forestry Research and Management 13: 31-47.

Borah N, Athokpam FD, Garkoti SC, Das AK \& Hore DK (2014) Structural and compositional variations in undisturbed and disturbed tropical forests of Bhuban hills in south Assam, India. International Journal of biodiversity sciences, ecosystem services and management 10(1): 9-19.

www.tropicalplantresearch.com 
Bourgeron PS (1983) "Spatial Aspects of Vegetation Structure". In Frank B. Golley. Tropical Rain Forest Ecosystems. Structure and Function. Ecosystems of the World (14A ed). Elsevier Scientific, Pp. 2947. [ISBN 0-444-41986-1]

Brashears MB, Fajvan MA \& Schuler TM (2004) An assessment of canopy stratification and tree species diversity following clear cutting in central Appalachian hardwoods. Forest Science 50(1): 54-64.

Cardinale BJ, Duffy JE, Gonzalez A, Hooper DU, Perrings C, Venail P, Narwani A, Mace GM, Tilman D, Wardle DA, Kinzig AP, Daily GC, Loreau M, Grace JB, Larigauderie A, Srivastava DS \& Naeem S (2012) Biodiversity loss and its impact on humanity. Nature 486(7401): 59-67.

Chadman S (2008) Defining degradation for an effective mechanism to reduce emissions from deforestation and forest degradation (REED). SBSTA Workshop on Forest Degradation (B0nn, 21-22 October, 2008)

Chavan BL \& Rasal GB (2010) Sequestred standing Carbon Stock in selective Tress species grown in University Campus at Aurangabad, Maharasgtra, India. International Journal of Engineering Science and Technology 2(7): 3003-3007.

Daniel TC, Andreas M, Arne A, Oliver A, James WB, Kai MA, Chan, Robert C, Thomas E, Courtney GF, Paul HG, Adrienne GR, Rebecca L, Susanne M, Marianne P, Robert G R, Thomas S, Thomas S, Ihor S, Marja S, Karolina T, Jordan T \& Andreas VD (2012) Contributions of cultural services to the ecosystem services agenda. Proceedings of the National Academy of Sciences 109(23): 8812-8819.

Dengler J (2017) Phytosociology. 1-6. 10.1002/9781118786352.wbieg0136.

Ekpo FE, Asuquo ME, \& Akpabio J (2011) Conserving Biological Diversity for Sustainable Uses in Tropical Rainforest of Nigeria. Journal of Environmental Issues and Agriculture in Developing Countries 3: 102109.

FAO (1988) Soil Map of the World, Revised Legend. World Soil Resources, Report 60. FAO, UNESCO, Rome.

FAO (2001) Forest Resources Assessment 2000. Food and Agriculture Organisation, Rome, Italy.

FAO (2006) Global forest resources assessment 2005: Progress towards sustainable forest management. Food and Agricultural Organization, Rome, Italy. Forestry paper 147: 348.

FAO (2011) Forest Carbon Partnership Fund, Readiness Preparation Proposal. Version 4 March 2011. Forest Administration. Phnom Penh.

Fasinmirin JT\& Oguntuase AM (2008) Soil moisture distribution pattern in Amaranthus cruentus field under drip irrigation system. African Journal of Agricultural Research 3(7): 486-493.

GOFC-GOLD (2009) Reducing greenhouse gas emissions from deforestation and degradation in developing countries: a sourcebook of methods and procedures for monitoring, measuring and reporting. GOFC-GOLD Report Version COP14-2 GOFC-GOLD Project Office, Natural Resources Canada, Alberta, Canada.

Guo Y, Gong P \& Amundson R (2003) Pedodiversity in the United States of America. Geoderma 117: 99-115.

Halperin JJ \& Turner RL (2013) Forest degradation in Cambodia: an assessment of monitoring options in the Central Cardamom Protected Forest. United States Forest Service International Programs, Washington, DC. pp. 8-18.

Huang WD, Pohlonen V, Johansson S, Nashanda M, Katigula MIL \& Luukkanen O (2003) Species diversity, forest structure and species composition in Tanzanian tropical forests. Forest Ecology and Management 173: $11-24$.

Husch B, Beers TW \& Keenshaw JA (2003) Forest Mensuration. JohnWiley \& Sons, Hoboken, NJ, USA, 4th edition, 2003.

Ifo SA, Jean-Marie M, Félix K, Joseph Y, Saint FN, Leslie NOB, Helischa Mampouya, Charlotte J, Yannick B, Alima BM, Mackline M, Dulsaint M, Roland O, Lenguiya RM, Yeto EMW, Brice CO, \& Loumeto JJ (2016) Tree Species Diversity, Richness, and Similarity in Intact and Degraded Forest in the Tropical Rainforest of the Congo Basin: Case of the Forest of Likouala in the Republic of Congo. International Journal of Forestry Research 2016: Article ID 7593681, 12 pages.

Isichei AO (1995) Omo biosphere reserve, current status, utilization of biological resources and sustainable management (Nigeria). UNESCO South-South cooperation programme working, pp. 11.

IUCN (2008) Guidelines on the management of protected areas. International Union for Conservation of Nature (IUCN), Gland, Switzerland and Cambridge document.

Kent M \& Coker P (1992) Vegetation description and analysis: A practical approach. Belhaven press, London, $363 \mathrm{p}$. 
Lafrankie JV, Ashton PS, Chuyong GB, Co L, Condit R, Davies SJ, Foster R, Hubbell SP, Kenfack D, Lagunzad D, Losos EC, Nor NSM, Tan S, Thomas DW, Valencia R \& Villa G (2006) Contrasting structure and composition of the understory in species-rich tropical rainforests. Ecology 87: 2298-2305.

Lu XT, Yin JX \& Tang JW (2010) Structure, tree species diversity and composition of tropical seasonal rainforests in Xishuangbanna, south-west China. Journal of Tropical Forest Science 22 (3): 260-270.

Mackey B, Heather K, Sandy B \& David L (2008) Green Carbon: The role of natural forests in carbon storage. Part 1. A green carbon account of Australia's south-eastern Eucalypt forests and policy implications Australian National University Press, Canberra; p 44; http://epress.anu.edu.au/green_carbon_citation.html

Okali, DUU \& Ola-Adams BA (1987): Tree Population Changes in Threatened Rainforest at Omo Forest Reserve, South West Nigeria. Journal of Tropical Ecology 3 (4): 291-314.

Onyekwelu JC, Adekunle VAJ \& Adeduntan SA (2005) Does the Tropical rainforest ecosystem possess the ability to recover from severe degradation? In: Popoola L, Mfon P \& Oni PI (eds) Sustainable forest management in Nigeria: lessons and prospects. Proceeding of the $30^{\text {th }}$ Annual conference of the Forestry Association of Nigeria, Kaduna, $07^{\text {th }}-11^{\text {th }}$ Nov. 2005, pp. 145-163.

Onyekwelu JC, Reinhard M \& Bernd S (2007) Tree species diversity and soil status of two natural forest ecosystems in lowland humid tropical rainforest region of Nigeria. Conference of International Agriculture Research for Development. Tropentag 2007, pp. 1-4.

Parthasarathy N (2001) Changes in forest composition and structure in three sites of tropical evergreen forest around Sengaltheri, Western Ghats. Current Science 80(3): 389-393.

Pelemo OJ, Adeofin CO, Osudiala CS \& Adetogun AC (2011) Assessment of growth dynamics of tree species in SNR2, Akure forest reserve, Nigeria. Journal of Research in Forestry, Wildlife and Environment 3(2): $39-45$.

Phillips O, Martinez RV, Vargas PN, Monteagudo AL, Zans MEC, Sanchez WG, Cruz AP, Timana M, YilHalla M \& Rose S (2003) .Efficient plot-base floristic assessment of tropical forests. Journal of Tropical Ecology 19: 629-645.

Price PW (1997) Insect Ecology, $3^{\text {rd }}$ edition. Wiley, NY, USA pp. 88.

Rajkumar M \& Parthasarathy N (2008) Tree Diversity and Structure of Andaman Giant Evergreen Forests, India. Taiwania 53(4): 356-368.

Royal Society (2003) Measuring Biodiversity for Conservation. Royal Society Policy Document (November 2003).

Sarkar M \& Devi A (2014) Assessment of diversity, population structure and regeneration status of tree species in Hollongapar Gibbon Wildlife Sanctuary, Assam, Northeast India. Tropical Plant Research 1(2): 26-36.

Sedano F, SilvaJ A, RMachoco, Meque, CH, Sitoe A, Ribeiro N, Anderson K, Ombe ZA, Baule SH \& Tucker CJ (2016) The impact of charcoal production on forest degradation: a case study in Tete, Mozambique. Environmental Research Letter 11(9): 13 p.

Simula Markku (2009) Towards defining degradation: Comparative analysis of existing definitions. Food and Agriculture Organization. Discuss paper, $59 \mathrm{p}$.

UNEP (2011) Towards a Green Economy: pathway to sustainable development and poverty eradication - a syntheses for policy makers. United Nations Environment Programme, World Conservation Monitoring Centre, Nairo Kenya.

van der Werf GR, Morton DC, DeFries RS, Giglio L, Randerson JT, Collatz GJ, \& Kasibhatla PS (2009) Estimates of fire emissions from an active deforestation region in the southern Amazon based on satellite data and biogeochemical modeling. Biogeosciences 6: 235-249.

Vinayaka KS \& Krishnamurthy YL (2016) Floristic composition and vegetation analysis of Hulikal Ghat region, central Western Ghats, Karnataka. Tropical Plant Research 3(3): 654-661. 